EPJ Web of Conferences 116, 01003 (2016)

DOI: $10.1051 /$ epjconf/201611601003

(C) Owned by the authors, published by EDP Sciences, 2016

\title{
The optical module of Baikal-GVD
}

A.D. Avrorin ${ }^{1}$, A.V. Avrorin ${ }^{1}$, V.M. Aynutdinov ${ }^{1}$, R. Bannash ${ }^{7}$, I.A. Belolaptikov ${ }^{2}$, D.Yu. Bogorodsky ${ }^{3}$, V.B. Brudanin ${ }^{2}$, N.M. Budnev ${ }^{3}$, I.A. Danilchenko ${ }^{1}$, G.V. Domogatsky ${ }^{1}$, A.A. Doroshenko ${ }^{1}$, A.N. Dyachok ${ }^{3}$, Zh.-A.M. Dzhilkibaev ${ }^{1}$, S.V. Fialkovsky ${ }^{5}$, A.R. Gafarov ${ }^{3}$, O.N. Gaponenko ${ }^{1}$, K.V. Golubkov' ${ }^{1}$, T.I. Gress ${ }^{3}$, Z. Honz ${ }^{2}$, K.G. Kebkal ${ }^{7}$, O.G. Kebkal ${ }^{7}$, K.V. Konischev², A.V. Korobchenko ${ }^{2}$, A.P. Koshechkin ${ }^{1}$, F.K. Koshel ${ }^{1}$, A.V. Kozhin ${ }^{4}$, V.F. Kulepov ${ }^{5}$, D.A. Kuleshov ${ }^{1}$, V.I. Ljashuk ${ }^{1}$, M.B. Milenin ${ }^{5}$, R.A. Mirgazov ${ }^{3}$, E.R. Osipova ${ }^{4}$, A.I. Panfilov ${ }^{1}$, L.V. Pan'kov ${ }^{3}$, E.N. Pliskovsky ${ }^{2}$, M.I. Rozanov ${ }^{6}$, E.V. Rjabov ${ }^{3}$, B.A. Shaybonov ${ }^{2}$, A.A. Sheifler ${ }^{1}$, M.D. Shelepov ${ }^{1}$, A.V. Skurihin ${ }^{4}$, A.A. Smagina ${ }^{2}$, O.V. Suvorova ${ }^{1}$, V.A. Tabolenko ${ }^{3}$, B.A. Tarashansky ${ }^{3}$, S.A. Yakovlev ${ }^{7}$, A.V. Zagorodnikov ${ }^{3}$, V.A. Zhukov ${ }^{1}$, and V.L. Zurbanov ${ }^{3}$

${ }^{1}$ Institute for Nuclear Research, 60th October Anniversary pr. 7A, Moscow 117312, Russia

2 Joint Institute for Nuclear Research, Dubna 141980, Russia

${ }^{3}$ Irkutsk State University, Irkutsk 664003, Russia

${ }^{4}$ Skobeltsyn Institute of Nuclear Physics MSU, Moscow 119991, Russia

${ }^{5}$ Nizhni Novgorod State Technical University, Nizhni Novgorod 603950, Russia

${ }^{6}$ St. Petersburg State Marine University, St. Petersburg 190008, Russia

${ }^{7}$ EvoLogics GmbH, Berlin, Germany

\begin{abstract}
The Baikal-GVD neutrino telescope in Lake Baikal is intended for studying astrophysical neutrino fluxes by recording the Cherenkov radiation of the secondary muons and showers generated in neutrino interactions. The first stage of Baikal-GVD will be equipped with about 2300 optical modules. We describe the design of the optical module, the front-end electronics and the laboratory characterization and calibration before deployment.
\end{abstract}

\section{Introduction}

The objective of the Baikal Project is the creation of a kilometer-scale high-energy neutrino observatory: the Gigaton Volume Detector (GVD) in Lake Baikal [1-4]. The first phase of GVD will consist of 12 independent (i.e. connected to the shore by 27 individual electro-optical cables) clusters of strings. In April 2015 the first cluster of Baikal-GVD was deployed in Lake Baikal and put into operation. It comprises a total of 192 optical modules (OM) arranged at eight $345 \mathrm{~m}$ long strings, as well as an acoustic positioning system. Seven side strings are located at $40 \mathrm{~m}$ distances from a central one. Each string comprises 24 OMs spaced by $15 \mathrm{~m}$ at depths of $900 \mathrm{~m}$ to $1250 \mathrm{~m}$ below the surface. An optical module contains a large area photomultiplier R7081-100 that detects Cherenkov radiation produced by relativistic charged particles passing through the water. The information from the ensemble of

This is an Open Access article distributed under the terms of the Creative Commons Attribution License 4.0, which permits unrestricted use, distribution, and reproduction in any medium, provided the original work is properly cited. 


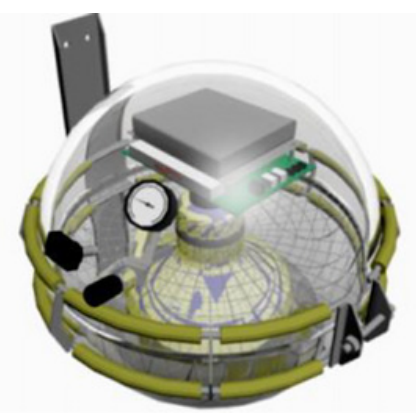

Figure 1. The Baikal-GVD optical module.

OMs allows reconstruction of direction and energy of the parent neutrino. Optical modules are the key elements of a neutrino telescope. The present paper presents the design and performance of the components of the optical module, and describes selected results of the tests of 87 OMs.

\section{The Optical Module Design}

A sketch of a GVD optical module is presented in Fig. 1. The OM contains a photomultiplier tube (PMT) enclosed in a transparent, nearly spherical pressure housing (VITROVEX) with $42 \mathrm{~cm}$ diameter. The optical contact between the photocathode region of the tube and the pressure sphere is provided by optically transparent silicone gel. A high permittivity alloy cage surrounds the PMT, shielding it against the Earth's magnetic field. A vacuum valve allows evacuating the sphere down to $0.7 \mathrm{~atm}$. The OM is equipped with one deep-underwater connector (SubConn Low Profile 5-contacts). It is used for analog pulse transmission, slow control (2-wire RS-485) and OM power supply (12 VDC). The OM electronics unit is mounted directly on the PMT base.

After testing different options for the photomultiplier, Hamamatsu R7081-100 was selected as a light sensor for the OM. This PMT has a hemispherical SBA (super bialkali) photocathode with 10" diameter and a quantum efficiency at peak up to $35 \%$.

The block diagram of the optical module electronics is presented in Fig. 2. The OM electronics includes a controller, a high voltage (HV) power supply unit, a fast two-channel amplifier, and a LED flasher. The OM controller is intended for communication to a Central electronics Module, for $\mathrm{HV}$ regulation and monitoring, for PMT noise measurements, and for time and amplitude calibration with LEDs. It is designed by SNIIP-AUNIS Ltd (Russia) on the basis of the SiLabs C8051F121 microcontroller.

Slow control data to and from the OMs are transferred via an underwater RS-485 bus. The unit has an instruction set for the control of OM electronics: HV control (to switch HV on/off, to set the PMT voltage and readout the HV value), LED flasher control (to set LED intensities, the delay between LED pulses, and the period of flashes), the counter control (setting counter threshold, time window, off-duty factor and the size of circular memory data buffer). Also a set of procedures is foreseen for the laboratory calibration of the counter threshold and PMT voltage.

The PMT power supply is provided by a passive HV divider circuit with a resistance of $18 \mathrm{M} \Omega$ and a HV unit (SHV 12-2.0 K 1000 P produced by TRACO Electronic AG) with positive polarity. The tube gains have been adjusted to about $10^{7}$.

The PMT amplifier is comprised of two channels. The first channel with an amplification factor of 14 forms a signal with negative polarity that is transmitted to the ADC board. The second channel with an amplification factor of 21 produces positive pulses that are intended for PMT noise monitoring. The 


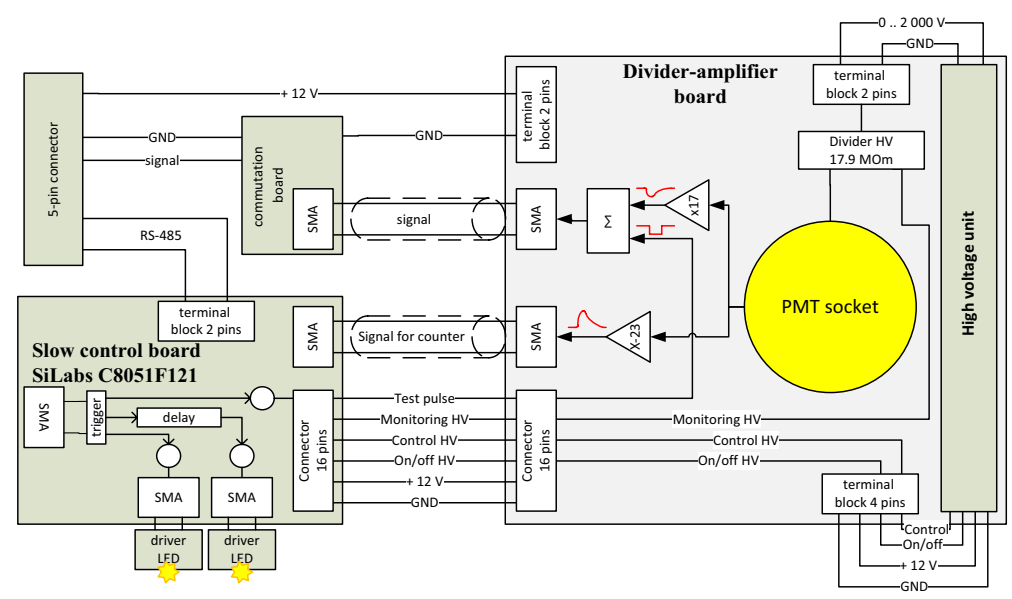

Figure 2. Block diagram of the OM electronics.

outer cascades are implemented as emitter-follower amplifiers and provide operation with $50 \Omega$ load. The maximum amplitude for both channels is limited to $4 \mathrm{~V}$.

The LED flasher is intended for time and amplitude calibration of OM channels during long-term exposition. It includes two LEDs Kingbright L7113 with a dominant wavelength about $470 \mathrm{~nm}$. The LED pulse has a width of $\sim 6 \mathrm{~ns}$ (FWHM). The control system of the flasher has to provide independent tuning of two LED luminosities in a wide dynamic range (up to about $10^{8}$ photons per LED flash) and has to have a minimal level of cross-talk between two LED channels (less than $1 \%$ ).

The OM counter with programmable threshold is intended for PMT count rate monitoring. Count rate data is accumulated in the circular buffer and transmitted to the shore for each OM.

\section{OMs calibration and characterization}

Before OM deployment in Lake Baikal a series of test procedures is foreseen. There are tests of all OM electronic components, stress tests, and check-up of the OM in various modes of operation (final OM tests before transportation to Baikal). Final OM tests are performed by means of a digital storage oscilloscope (LeCroy HDO 4034, $350 \mathrm{MHz}$ bandwidth, 0.4 ns samples, 4 channels). Four OMs are housed in a screened dark box and are connected to the oscilloscope inputs with $90 \mathrm{~m}$ coaxial cables, identical to the underwater OM cables. The test procedures are performed after at least 2 hours exposition in the darkness with PMT high voltage switched on. Internal LEDs of the OMs are used as calibration light sources. The OM test procedures are fully automatized and comprise a set of measurements of the time and amplitude parameters of the OMs.

The first stage of the OM test procedure is an adjustment of the PMT power supply voltages to provide OM channel gains about $10^{8}$. An OM analog channel comprises PMT, preamplifier and $90 \mathrm{~m}$ coaxial cable connecting OM and Central electronics Module. Taking into account signal amplification with the preamplifier $\left(\mathrm{k}_{\mathrm{amp}}=14\right)$ and pulse attenuation in the cable $\left(\mathrm{k}_{\mathrm{att}}=0.7\right)$, a $10^{8}$ channel gain corresponds to a PMT gain about $10^{7}$.

The OM channel gains were derived on the basis of the single photoelectron distributions (SPE spectra) of the PMTs obtained with LED sources. Intensities of the LEDs were adjusted to provide a detection probability of SPE signals of $10 \%$. The SPE pulse detection thresholds were about 0.2 SPE pulse amplitude. The oscilloscope input was triggered by the synchronization signal of the LED pulse generator. The check for pedestal and noise contributions was done with the LED light output disabled. 

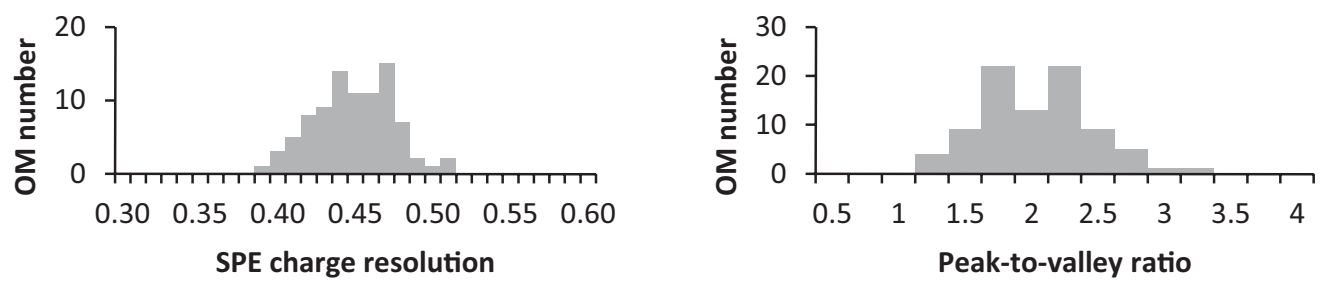

Figure 3. The distributions of the OMs on SPE charge resolution (left) and on the peak-to-valley ratio (right).
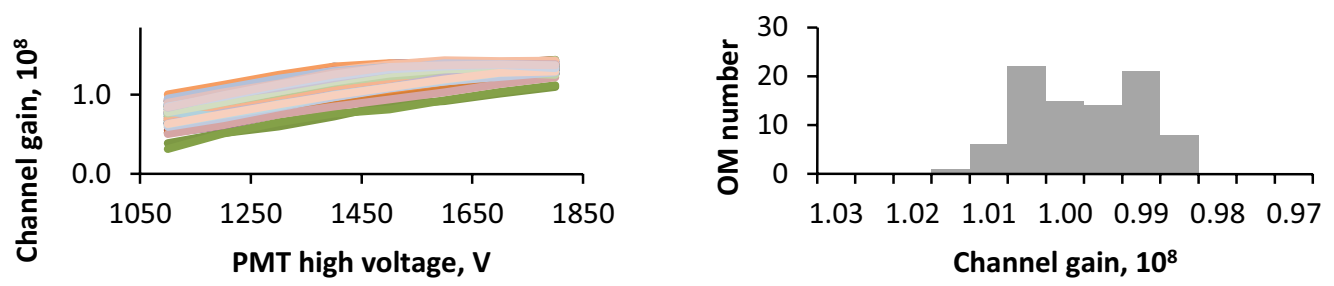

Figure 4. The dependences of the channel gains on high voltage for the set of $87 \mathrm{OMs}$ (left), and OMs distribution on the fitted channel gain (right).
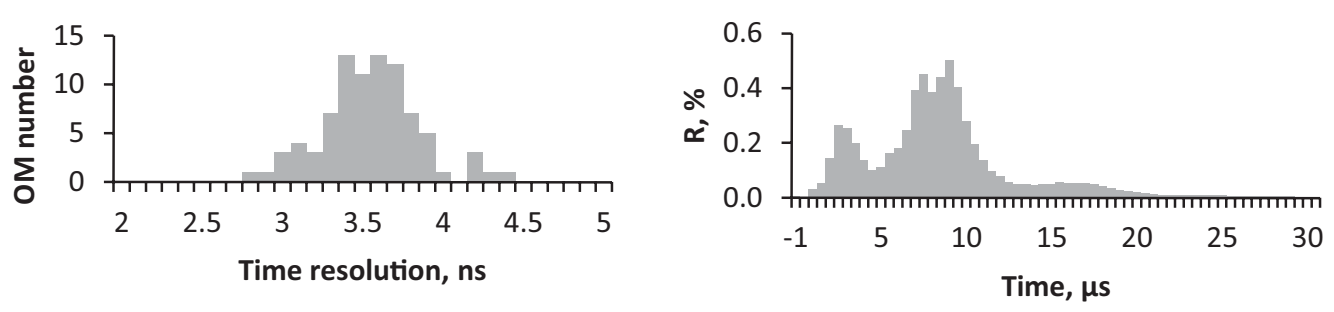

Figure 5. The distribution of the OMs on the time resolution (left) and the typical time dependence of the afterpulse charges for primary pulses with one photoelectron (right).

The distributions of the OMs on SPE charge resolution (left) and on the peak-to-valley ratio are presented in Fig. 3. The SPE charge resolution is defined as one standard deviation of the SPE spectrum. The contribution of multi-electron pulses in the SPE spectrum overestimates the SPE charge resolution by about $20 \%$.

The results of the high voltage adjustment are presented in the Fig. 4. The channel gain of $10^{8}$ is provided by divider voltages between $1150 \mathrm{~V}$ and $1750 \mathrm{~V}$ for the investigated set of PMTs. Adjusted channel gains are about $1 \times 10^{8}$.

The OM time resolution (the standard deviations of hit times of the SPE pulses) is presented in Fig. 5 (left). Afterpulse measurements were made with LED pulses of about $5 \mathrm{~ns}$ width. The total charge of the afterpulses $\left(\mathrm{N}_{\mathrm{pe}}\right.$ afterpulses) was measured in the range from $300 \mathrm{~ns}$ up to $30 \mu \mathrm{s}$ after the main pulse, in steps of $500 \mathrm{~ns}$ and for various LED intensities. $\mathrm{N}_{\mathrm{pe}}$ afterpulses grows almost linearly with the flash brightness. We characterize the OM afterpulses by the parameter $\mathrm{R}=\mathrm{N}_{\text {pe afterpulses }} / \mathrm{N}_{\text {pe main pulse }} \times 100 \%$. The typical dependence of $\mathrm{R}$ on the time after the main pulse is presented in Fig. 5 (right). For most of the OMs, the integral charges of the afterpulses correspond to about $0.10 \ldots 0.15 \mathrm{SPE}$ per primary photoelectron. 


\section{Conclusion}

For the OM mass production a fully automatized test facility was designed. The test of the OM performance with this facility provides information about the basic OM parameters: SPE spectrum, time resolution, systematic shifts of the hit time in dependence on the pulse amplitude, saturation curve, and afterpulse probability. These data are used as input for the detailed simulation of the Baikal-GVD physics events.

This work was supported by the Russian Found for Basic Research (grants 13-02-12221, 14-02-00175, 14-0200972).

\section{References}

[1] A. Avrorin et al., The prototyping/early construction phase of the BAIKAL-GVD project, NIM A742, 82

[2] V. Aynutdinov et al., The prototype string for the km3-scale Baikal neutrino telescope, NIM A602, 227

[3] V. Aynutdinov et al., The gigaton volume detector in Lake Baikal, NIM A639, 30

[4] A. Avrorin et al., Status and recent results of the Baikal-GVD project, Phys. of Part. and Nucl. 46, 211 